JOURNAL OF

FUNCTION SPACES AND APPLICATIONS

Volume 6, Number 3 (2008), 277-292
(C) $200^{*}$, Scientific Horizon http://www.jfsa.net

\title{
A criterion of weak compactness for operators on subspaces of Orlicz spaces
}

\author{
Pascal Lefèvre, Daniel Li,
}

Hervé Queffélec and Luis Rodríguez-Piazza

(Communicated by Nigel Kalton)

2000 Mathematics Subject Classification. Primary: 46E30; Secondary: 46B20.

Keywords and phrases. Morse-Transue space, Orlicz space, weakly compact operators.

Abstract. We give a criterion of weak compactness for the operators on the Morse-Transue space $M^{\Psi}$, the subspace of the Orlicz space $L^{\Psi}$ generated by $L^{\infty}$.

\section{Introduction and Notation}

In 1975, C. Niculescu established a characterization of weakly compact operators $T$ from $\mathcal{C}(S)$, where $S$ is a compact space, into a Banach space $Z$ ([14, 15], see [3] Theorem 15.2 too): $T: \mathcal{C}(S) \rightarrow Z$ is weakly compact if and only if there exists a Borel probability measure $\mu$ on $S$ such that, for every $\epsilon>0$, there exists a constant $C(\epsilon)>0$ such that:

$$
\|T f\| \leq C(\epsilon)\|f\|_{L^{1}(\mu)}+\epsilon\|f\|_{\infty}, \quad \forall f \in \mathcal{C}(S) .
$$

The same kind of result was proved by $\mathrm{H}$. Jarchow for $\mathbb{C}^{*}$-algebras in [7], and by the first author for $A(\mathbb{D})$ and $H^{\infty}$ (see [11]). The criterion for $H^{\infty}$ 
played a key role to give an elementary proof of the equivalence between weak compactness and compactness for composition operators on $H^{\infty}$.

Beside these spaces, one natural class of Banach spaces is the class of Orlicz spaces $L^{\Psi}$. Unfortunately, we shall see that the above criterion is in general not true for Orlicz spaces. However, it remains true when we restrict ourselves to subspaces of the Morse-Transue space $M^{\Psi}$. This space is the closure of $L^{\infty}$ in the Orlicz space $L^{\Psi}$.

In this paper, we first give a characterization of the operators from a subspace of $M^{\Psi}$ which fix no copy of $c_{0}$. When the complementary function of $\Psi$ satisfies $\Delta_{2}$, that gives a criterion of weak compactness. If moreover $\Psi$ satisfies a growth condition, that we call $\Delta^{0}$, the criterion has a more usable formulation, analogous to those described above.

As in the case of $H^{\infty}$ (but this is far less elementary), this new version obtained for subspaces of Morse-Transue spaces (Theorem 4), combined with a study of generalized Carleson measures, may be used to prove the equivalence between weak compactness and compactness for composition operators on Hardy-Orlicz spaces (see [13]), when $\Psi$ satisfies $\Delta^{0}$.

However, we think that this characterization has an intrinsic interest for Orlicz spaces, and will be useful not only for composition operators (see Remark 5 at the end of the paper).

In this note, we shall consider Orlicz spaces defined on a probability space $(\Omega, \mathbb{P})$, that we shall assume non purely atomic.

By an Orlicz function, we shall understand that $\Psi:[0, \infty] \rightarrow[0, \infty]$ is a non-decreasing convex function such that $\Psi(0)=0$ and $\Psi(\infty)=\infty$. To avoid pathologies, we shall assume that we work with an Orlicz function $\Psi$ having the following additional properties: $\Psi$ is continuous at 0 , strictly convex (hence strictly increasing), and such that

$$
\frac{\Psi(x)}{x} \underset{x \rightarrow \infty}{\longrightarrow} \infty
$$

This is essentially to exclude the case of $\Psi(x)=a x$. The Orlicz space $L^{\Psi}(\Omega)$ is the space of all (equivalence classes of) measurable functions $f: \Omega \rightarrow \mathbb{C}$ for which there is a constant $C>0$ such that

$$
\int_{\Omega} \Psi\left(\frac{|f(t)|}{C}\right) d \mathbb{P}(t)<+\infty
$$

and then $\|f\|_{\Psi}$ (the Luxemburg norm) is the infimum of all possible constants $C$ such that this integral is $\leq 1$. 
To every Orlicz function is associated the complementary Orlicz function $\Phi=\Psi^{*}:[0, \infty] \rightarrow[0, \infty]$ defined by:

$$
\Phi(x)=\sup _{y \geq 0}(x y-\Psi(y)) .
$$

The extra assumptions on $\Psi$ ensure that $\Phi$ is itself strictly convex.

Throughout this paper, we shall assume, except explicit mention of the contrary, that the complementary Orlicz function satisfies the $\Delta_{2}$ condition $\left(\Phi \in \Delta_{2}\right)$, i.e., for some constant $K>0$, and some $x_{0}>0$, we have:

$$
\Phi(2 x) \leq K \Phi(x), \quad \forall x \geq x_{0} .
$$

This is usually expressed by saying that $\Psi$ satisfies the $\nabla_{2}$ condition $\left(\Psi \in \nabla_{2}\right)$. This is equivalent to say that for some $\beta>1$ and $x_{0}>0$, one has $\Psi(x) \leq \Psi(\beta x) /(2 \beta)$ for $x \geq x_{0}$, and that implies that $\frac{\Psi(x)}{x} \underset{x \rightarrow \infty}{\longrightarrow} \infty$. In particular, this excludes the case $L^{\Psi}=L^{1}$.

When $\Phi$ satisfies the $\Delta_{2}$ condition, $L^{\Psi}$ is the dual space of $L^{\Phi}$.

We shall denote by $M^{\Psi}$ the closure of $L^{\infty}$ in $L^{\Psi}$. Equivalently (see [16], page 75$), M^{\Psi}$ is the space of (classes of) functions such that:

$$
\int_{\Omega} \Psi\left(\frac{|f(t)|}{C}\right) d \mathbb{P}(t)<+\infty, \quad \forall C>0 .
$$

This space is the Morse-Transue space associated to $\Psi$, and $\left(M^{\Psi}\right)^{*}=L^{\Phi}$, isometrically if $L^{\Phi}$ is provided with the Orlicz norm, and isomorphically if it is equipped with the Luxemburg norm (see [16], Chapter IV, Theorem 1.7 , page 110).

We have $M^{\Psi}=L^{\Psi}$ if and only if $\Psi$ satisfies the $\Delta_{2}$ condition, and $L^{\Psi}$ is reflexive if and only if both $\Psi$ and $\Phi$ satisfy the $\Delta_{2}$ condition. When the complementary function $\Phi=\Psi^{*}$ of $\Psi$ satisfies it (but $\Psi$ does not satisfy this $\Delta_{2}$ condition, to exclude the reflexive case), we have (see [16], Chapter IV, Proposition 2.8, page 122, and Theorem 2.11, page 123):

$$
\left(L^{\Psi}\right)^{*}=\left(M^{\Psi}\right)^{*} \oplus_{1}\left(M^{\Psi}\right)^{\perp},
$$

or, equivalently, $\left(L^{\Psi}\right)^{*}=L^{\Phi} \oplus_{1}\left(M^{\Psi}\right)^{\perp}$, isometrically, with the Orlicz norm on $L^{\Phi}$.

For all the matter about Orlicz functions and Orlicz spaces, we refer to [16], or to [9]. 


\section{Main result}

Our goal in this section is the following criterion of weak compactness for operators. We begin with:

Theorem 1. Let $\Psi$ be an arbitrary Orlicz function, and let $X$ be a subspace of the Morse-Transue space $M^{\Psi}$. Then an operator $T: X \rightarrow Y$ from $X$ into a Banach space $Y$ fixes no copy of $c_{0}$ if and only if for each $\varepsilon>0$, there exists $C_{\varepsilon}>0$ such that

$$
\|T f\| \leq\left[C_{\varepsilon} \int_{\Omega} \Psi\left(\varepsilon \frac{|f|}{\|f\|_{\Psi}}\right) d \mathbb{P}+\varepsilon\right]\|f\|_{\Psi}, \quad \forall f \in X .
$$

Recall that saying that $T$ fixes a copy of $c_{0}$ means that there exists a subspace $X_{0}$ of $X$ isomorphic to $c_{0}$ such that $T$ realizes an isomorphism between $X_{0}$ and $T\left(X_{0}\right)$.

Before proving that, we shall give some consequences. First, we have

Corollary 2. Assume that the complementary function of $\Psi$ has $\Delta_{2}$ $\left(\Psi \in \nabla_{2}\right)$. Then for every subspace $X$ of $M^{\Psi}$, and every operator $T: X \rightarrow Y, T$ is weakly compact if and only if it satisfies (1).

Proof. When the complementary function of $\Psi$ has $\Delta_{2}$, one has the decomposition (*), which means that $M^{\Psi}$ is $M$-ideal in its bidual (see [6, Chapter III]); this result was first shown by D. Werner [17] (see also [6, Chapter III, Example 1.4 (d), page 105]) by a different way, using the ball intersection property; note that in these references, it is moreover assumed that $\Psi$ does not satisfy the $\Delta_{2}$ condition, but if it satisfies it, the space $L^{\Psi}$ is reflexive, and so the result is obvious. But every subspace $X$ of a Banach space which is $M$-ideal of its bidual has Pełczyńki's property $(V)$ $([4,5]$; see also [6], Chapter III, Theorem 3.4), which means that operators from $X$ are weakly compact if and only if they fix no copy of $c_{0}$.

With $\Psi$ satisfying the following growth condition, the characterization (1) takes on a more usable form.

Definition 3. We say that the Orlicz function $\Psi$ satisfies the $\Delta^{0}$ condition if for some $\beta>1$

$$
\lim _{x \rightarrow+\infty} \frac{\Psi(\beta x)}{\Psi(x)}=+\infty .
$$

This growth condition is a strong negation of the $\Delta_{2}$ condition and it implies that the complementary function $\Phi=\Psi^{*}$ of $\Psi$ satisfies the $\Delta_{2}$ condition.

Note that in the following theorem, we cannot content ourselves with $\Psi \notin \Delta_{2}$ (i.e. $\left.\lim \sup _{x \rightarrow+\infty} \Psi(\beta x) / \Psi(x)=+\infty\right)$, instead of $\Psi \in \Delta^{0}$ (see 
Remark 3 in Section 3). An interesting question is whether the condition $\Psi \in \Delta^{0}$ is actually necessary for this characteriztion.

Theorem 4. Assume that $\Psi$ satisfies the $\Delta^{0}$ condition, and let $X$ be a subspace of $M^{\Psi}$. Then every linear operator $T$ mapping $X$ into some Banach space $Y$ is weakly compact if and only if for some (and then for all) $1 \leq p<\infty$ and all $\varepsilon>0$, there exists $C_{\varepsilon}>0$ such that

$$
\|T(f)\| \leq C_{\varepsilon}\|f\|_{p}+\varepsilon\|f\|_{\Psi}, \quad \forall f \in X .
$$

Remark 5. This theorem extends [12] Theorem II.1. As in the case of $\mathbb{C}^{*}$-algebras (see [3], Notes and Remarks, Chap. 15), there are miscellaneous applications of such a characterization.

Remark 6. Contrary to the $\Delta_{2}$ condition where the constant 2 may be replaced by any constant $\beta>1$, in this $\Delta^{0}$ condition, the constant $\beta$ cannot be replaced by another, as the following example shows.

Example 7. There exists an Orlicz function $\Psi$ such that

$$
\lim _{x \rightarrow+\infty} \frac{\Psi(5 x)}{\Psi(x)}=+\infty
$$

but

$$
\liminf _{x \rightarrow+\infty} \frac{\Psi(2 x)}{\Psi(x)}<+\infty .
$$

Indeed, let $\left(c_{n}\right)_{n}$ be an increasing sequence of positive numbers such that $\lim _{n \rightarrow \infty} \frac{c_{n+1}}{c_{n}}=+\infty$, take $\psi(t)=c_{n}$ for $t \in\left(4^{n}, 4^{n+1}\right]$ and $\Psi(x)=\int_{0}^{x} \psi(t) d t$. Then (2) is verified. On the other hand, if $x_{n}=2 \cdot 4^{n}$, one has $\Psi\left(x_{n}\right) \geq$ $c_{n} 4^{n}$, and $\Psi\left(2 x_{n}\right) \leq c_{n} 4^{n+1}$, so we get (3).

Before proving Theorem 4, let us note that it has the following straightforward corollary.

Corollary 8. Let $X$ be like in Theorem 4 , and assume that $\mathcal{F}$ is a family of operators from $X$ into a Banach space $Y$ with the following property: there exists a bounded sequence $\left(g_{n}\right)_{n}$ in $X$ such that $\lim _{n \rightarrow \infty}\left\|g_{n}\right\|_{1}=0$ and such that an operator $T \in \mathcal{F}$ is compact whenever

$$
\lim _{n \rightarrow \infty}\left\|T g_{n}\right\|=0 \text {. }
$$

Then every weakly compact operator in $T \in \mathcal{F}$ is actually compact.

In the forthcoming paper [13], we prove, using a generalization of the notion of Carleson measure, that a composition operator $C_{\phi}: H^{\Psi} \rightarrow H^{\Psi}$ 
$\left(H^{\Psi}\right.$ is the space of analytic functions on the unit disk $\mathbb{D}$ of the complex plane whose boundary values are in $L^{\Psi}(\partial \mathbb{D})$, and $\phi: \mathbb{D} \rightarrow \mathbb{D}$ is an analytic self-map) is compact whenever

$$
\lim _{r \rightarrow 1^{-}} \sup _{|\xi|=1} \Psi^{-1}(1 /(1-r))\left\|C_{\phi}\left(u_{\xi, r}\right)\right\|_{\Psi}=0
$$

where

and we have

$$
u_{\xi, r}(z)=\left(\frac{1-r}{1-\bar{\xi} r z}\right)^{2}, \quad|z|<1
$$

$$
\lim _{r \rightarrow 1^{-}} \sup _{|\xi|=1} \Psi^{-1}(1 /(1-r))\left\|C_{\phi}\left(u_{\xi, r}\right)\right\|_{\Psi}=0
$$

when $C_{\phi}$ is weakly compact and $\Psi \in \Delta^{0}$.

Though the situation does not fit exactly as in Corollary 8 (not because of the space $H^{\Psi}$, which is not a subspace of $M^{\Psi}$ : we actually work in $H M^{\Psi}=H^{\Psi} \cap M^{\Psi}$ since $u_{\xi, r} \in H M^{\Psi}$, but because of the fact that we ask a uniform limit for $|\xi|=1$ ), the same ideas allow us to get, when $\Psi$ satisfies the condition $\Delta^{0}$, that $C_{\phi}$ is compact if and only if it is weakly compact.

Proof of Theorem 4. Assume that we have (W). We may assume that $p>1$, since if $(\mathrm{W})$ is satisfied for some $p \geq 1$, it is satisfied for

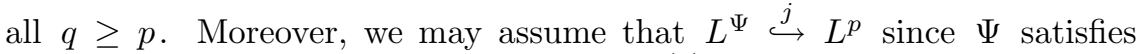
condition $\Delta^{0}$ (since we have: $\lim _{x \rightarrow+\infty} \frac{\Psi(x)}{x^{r}}=+\infty$, for every $r>0$ ). Then $T\left[\left(1 / C_{\varepsilon}\right) B_{L^{p}} \cap(1 / \varepsilon) B_{X}\right] \subseteq 2 B_{Y}$. Taking the polar of these sets, we get $T^{*}\left(B_{Y^{*}}\right) \subseteq\left(2 C_{\varepsilon}\right) B_{j^{*}\left[\left(L^{p}\right)^{*}\right]}+(2 \varepsilon) B_{X^{*}}$, for every $\varepsilon>0$. By a wellknown lemma of Grothendieck, we get, since $B_{j^{*}\left[\left(L^{p}\right)^{*}\right]}$ is weakly compact, that $T^{*}\left(B_{Y^{*}}\right)$ is relatively weakly compact, i.e. $T^{*}$, and hence also $T$, is weakly compact.

Conversely, assume in Theorem 4 that $T$ is weakly compact. We are going to show that $(\mathrm{W})$ is satisfied with $p=1$ (hence for all finite $p \geq 1$ ). Let $\varepsilon>0$. Since the $\Delta^{0}$ condition implies that the complementary function of $\Psi$ satisfies the $\Delta_{2}$ condition, Corollary 2 implies that, when $\|f\|_{\Psi}=1$

$$
\|T f\| \leq C_{\varepsilon / 2} \int_{\Omega} \Psi((\varepsilon / 2)|f|) d \mathbb{P}+\varepsilon / 2 .
$$

As $\Psi$ satisfies the $\Delta^{0}$ condition, there is some $\beta>1$ such that $\frac{\Psi(x)}{\Psi(\beta x)} \rightarrow 0$ as $x \rightarrow \infty$; hence, with $\kappa=\varepsilon / 2 C_{\varepsilon / 2}$, there exists some $x_{\kappa}>0$ such that $\Psi(x) \leq \kappa \Psi(\beta x)$ for $x \geq x_{\kappa}$. By the convexity of $\Psi$, one has 
$\Psi(x) \leq \frac{\Psi\left(x_{\kappa}\right)}{x_{\kappa}} x=: K_{\kappa} x$ for $0 \leq x \leq x_{\kappa}$. Hence, for every $x \geq 0$

$\Psi(x) \leq \kappa \Psi(\beta x)+K_{\kappa} x$. It follows that, for $f \in X$, with $\|f\|_{\Psi}=1$

$$
\int_{\Omega} \Psi((\varepsilon / 2)|f|) d \mathbb{P} \leq \kappa \int_{\Omega} \Psi(\beta(\varepsilon / 2)|f|) d \mathbb{P}+K_{\kappa} \frac{\varepsilon}{2}\|f\|_{1} \leq \kappa+K_{\kappa} \frac{\varepsilon}{2}\|f\|_{1}
$$

if we have chosen $\varepsilon \leq 2 / \beta$. Hence

$$
\|T f\| \leq C_{\varepsilon / 2}\left(\kappa+K_{\kappa} \frac{\varepsilon}{2}\|f\|_{1}\right)+\frac{\varepsilon}{2}=C_{\varepsilon / 2} \frac{\varepsilon}{2} K_{\kappa}\|f\|_{1}+\left(C_{\varepsilon / 2} \kappa+\frac{\varepsilon}{2}\right)=C_{\varepsilon}^{\prime}\|f\|_{1}+\varepsilon,
$$

which is $(\mathrm{W})$.

Remark 9. The sufficient condition is actually a general fact, which is surely well known (see [11], Theorem 1.1, for a similar result, and [3], Theorem 15.2 for $\mathcal{C}(K)$; see also [8], page 81), and has close connection with interpolation (see [2], Proposition 1), but we have found no reference, and so we shall state it separately without proof (the proof follows that given in [3], page 310).

Proposition 10. Let $T: X \rightarrow Y$ be an operator between two Banach spaces. Assume that there is a Banach space $Z$ and a weakly compact map $j: X \rightarrow Z$ such that: for every $\varepsilon>0$, there exists $C_{\varepsilon}>0$ such that

$$
\|T x\| \leq C_{\varepsilon}\|j x\|_{Z}+\varepsilon\|x\|_{X}, \quad x \in X .
$$

Then $T$ is weakly compact.

Note that, by the Davis-Figiel-Johnson-Pełczyński factorization theorem, we may assume that $Z$ is reflexive. We may also assume that $j$ is injective, because $\operatorname{ker} j \subseteq \operatorname{ker} T$, so $T$ induces a map $\tilde{T}: X / \operatorname{ker} j \rightarrow Y$ with the same property as $T$. Indeed, if $j x=0$, then $\|T x\| \leq \varepsilon\|x\|$ for every $\varepsilon>0$, and hence $T x=0$.

Proof of Theorem 1. Assume first that $T$ fixes a copy of $c_{0}$. There are hence some $\delta>0$ and a sequence $\left(f_{n}\right)_{n}$ in $X$ equivalent to the canonical basis of $c_{0}$ such that $\left\|f_{n}\right\|_{\Psi}=1$ and $\left\|T f_{n}\right\| \geq \delta$. In particular, there is some $M>0$ such that, for every choice of $\varepsilon_{n}= \pm 1$

$$
\left\|\sum_{n=1}^{N} \varepsilon_{n} f_{n}\right\|_{\Psi} \leq M, \quad N \geq 1 .
$$

Let $\left(r_{n}\right)_{n}$ be a Rademacher sequence. We have, first by Khintchine's inequality, then by Jensen's inequality and Fubini's Theorem 


$$
\begin{aligned}
\int_{\Omega} \Psi\left(\frac{1}{M \sqrt{2}}\left(\sum_{n=1}^{N}\left|f_{n}\right|^{2}\right)^{1 / 2}\right) d \mathbb{P} & \leq \int_{\Omega} \Psi\left[\frac{1}{M} \int_{0}^{1}\left|\sum_{n=1}^{N} r_{n}(t) f_{n}\right| d t\right] d \mathbb{P} \\
& \leq \int_{\Omega} \int_{0}^{1} \Psi\left[\frac{1}{M}\left|\sum_{n=1}^{N} r_{n}(t) f_{n}\right| d t\right] d \mathbb{P} \\
& =\int_{0}^{1} \int_{\Omega} \Psi\left[\frac{1}{M}\left|\sum_{n=1}^{N} r_{n}(t) f_{n}\right| d \mathbb{P}\right] d t \leq 1
\end{aligned}
$$

The monotone convergence Theorem gives then

$$
\int_{\Omega} \Psi\left(\frac{1}{M \sqrt{2}}\left(\sum_{n=1}^{\infty}\left|f_{n}\right|^{2}\right)^{1 / 2}\right) d \mathbb{P} \leq 1 .
$$

In particular, $\sum_{n=1}^{\infty}\left|f_{n}\right|^{2}$ is finite almost everywhere, and hence $f_{n} \rightarrow 0$ almost everywhere. Since $\Psi\left(\frac{1}{M \sqrt{2}}\left(\sum_{n=1}^{\infty}\left|f_{n}\right|^{2}\right)^{1 / 2}\right) \in L^{1}$, by the above inequalities, Lebesgue's dominated convergence Theorem gives

$$
\int_{\Omega} \Psi\left(\frac{\left|f_{n}\right|}{M \sqrt{2}}\right) d \mathbb{P} \underset{n \rightarrow \infty}{\longrightarrow} 0 .
$$

But that contradicts (1) with $\varepsilon \leq 1 / M \sqrt{2}$ and $\varepsilon<\delta$, since $\left\|T f_{n}\right\| \geq \delta$.

The converse follows from the following lemma.

Lemma 11. Let $X$ be a subspace of $M^{\Psi}$, and let $\left(h_{n}\right)_{n}$ be a sequence in $X$, with $\left\|h_{n}\right\|_{\Psi}=1$ for all $n \geq 1$, and such that, for some $M>0$

$$
\int_{\Omega} \Psi\left(\left|h_{n}\right| / M\right) d \mathbb{P} \underset{n \rightarrow \infty}{\longrightarrow} 0 .
$$

Then $\left(h_{n}\right)_{n}$ has a subsequence equivalent to the canonical basis of $c_{0}$.

Indeed, if condition (1) is not satisfied, there exist some $\varepsilon_{0}>0$ and functions $h_{n} \in X$ with $\left\|h_{n}\right\|_{\Psi}=1$ such that $\left\|T h_{n}\right\| \geq 2^{n} \int_{\Omega} \Psi\left(\varepsilon_{0}\left|h_{n}\right|\right) d \mathbb{P}+$ $\varepsilon_{0}$. That implies that $\int_{\Omega} \Psi\left(\varepsilon_{0}\left|h_{n}\right|\right) d \mathbb{P}$ tends to 0 , so Lemma 11 ensures that $\left(h_{n}\right)_{n}$ has a subsequence, which we shall continue to denote by $\left(h_{n}\right)_{n}$, equivalent to the canonical basis of $c_{0}$. Then $\left(T h_{n}\right)_{n}$ is weakly unconditionally Cauchy. Since $\left\|T h_{n}\right\| \geq \varepsilon_{0},\left(T h_{n}\right)_{n}$ has, by BessagaPełczyński's Theorem, a further subsequence equivalent to the canonical basis of $c_{0}$. It is then obvious that $T$ realizes an isomorphism between the spaces generated by these subsequences. 
Proof of Lemma 11. The proof uses the idea of the construction made in the proof of Theorem II.1 in [12], which it generalizes, but with some additional details.

By the continuity of $\Psi$, there exists $a>0$ such that $\Psi(a)=1$. Then, since $\Psi$ is increasing, we have, for every $g \in L^{\infty}, \int_{\Omega} \Psi\left(a \frac{|g|}{\|g\|_{\infty}}\right) d \mathbb{P} \leq 1$, and so $\|g\|_{\Psi} \leq(1 / a)\|g\|_{\infty}$. Now, choose, for every $n \geq 1$, positive numbers $\alpha_{n}<a / 2^{n+2}$ such that $\Psi\left(\alpha_{n} / 2 M\right) \leq 1$.

We are going to construct inductively a subsequence $\left(f_{n}\right)_{n}$ of $\left(h_{n}\right)_{n}$, a sequence of functions $g_{n} \in L^{\infty}$ and two sequences of positive numbers $\beta_{n}$ and $\varepsilon_{n} \leq \min \left\{1 / 2^{n+1}, M / 2^{n+1}\right\}$, such that, for every $n \geq 1$

(i) if we set $M_{1}=1$ and, for $n \geq 2$

$$
M_{n}=\max \left\{1, \Psi\left(\frac{\left\|g_{1}\right\|_{\infty}+\cdots+\left\|g_{n-1}\right\|_{\infty}}{2 M}\right)\right\}
$$

then $M_{n} \beta_{n} \leq 1 / 2^{n+1} ;$

(ii) $\left\|f_{n}\right\|_{\Psi}=1$;

(iii) $\left\|f_{n}-g_{n}\right\|_{\Psi} \leq \varepsilon_{n}$, with $\varepsilon_{n}$ such that $\beta_{n} \Psi\left(\alpha_{n} / 2 \varepsilon_{n}\right) \geq 2$;

(iv) $\mathbb{P}\left(\left\{\left|g_{n}\right|>\alpha_{n}\right\}\right) \leq \beta_{n}$;

(v) $\left\|\breve{g}_{n}\right\|_{\Psi} \geq 1 / 2$, with $\breve{g}_{n}=g_{n} \mathbb{I}_{\left\{\left|g_{n}\right|>\alpha_{n}\right\}}$.

We shall give only the inductive step, since the starting one unfolds identically. Suppose hence that the functions $f_{1}, \ldots, f_{n-1}, g_{1}, \ldots, g_{n-1}$ and the numbers $\beta_{1}, \ldots, \beta_{n-1}$ and $\varepsilon_{1}, \ldots, \varepsilon_{n-1}$ have been constructed. Choose then $\beta_{n}>0$ such that $M_{n} \beta_{n} \leq 1 / 2^{n+1}$. Note that $M_{n} \geq 1$ implies that $\beta_{n} \leq 1 / 2^{n+1}$. Since $\int_{\Omega} \Psi\left(\left|h_{k}\right| / M\right) d \mathbb{P} \rightarrow 0$ as $n \rightarrow \infty$, we can find $f_{n}=h_{k_{n}}$ such that $\left\|f_{n}\right\|_{\Psi}=1$, and moreover

$$
\mathbb{P}\left(\left\{\left|f_{n}\right|>\alpha_{n} / 2\right\}\right) \leq \frac{1}{\Psi\left(\alpha_{n} / 2 M\right)} \int_{\Omega} \Psi\left(\frac{\left|f_{n}\right|}{M}\right) d \mathbb{P} \leq \frac{\beta_{n}}{2} .
$$

Take now $\varepsilon_{n} \leq \min \left\{1 / 2^{n+1}, M / 2^{n+1}\right\}$ such that $0<\varepsilon_{n} \leq \alpha_{n} / 2 \Psi^{-1}\left(2 / \beta_{n}\right)$ and $g_{n} \in L^{\infty}$ such that $\left\|f_{n}-g_{n}\right\|_{\Psi} \leq \varepsilon_{n}$. Then, since

$$
\mathbb{P}\left(\left\{\left|f_{n}-g_{n}\right|>\alpha_{n} / 2\right\}\right) \Psi\left(\frac{\alpha_{n}}{2 \varepsilon_{n}}\right) \leq \int_{\Omega} \Psi\left(\frac{\left|f_{n}-g_{n}\right|}{\varepsilon_{n}}\right) d \mathbb{P} \leq 1,
$$

we have

$$
\begin{aligned}
\mathbb{P}\left(\left\{\left|g_{n}\right|>\alpha_{n}\right\}\right) & \leq \mathbb{P}\left(\left\{\left|f_{n}\right|>\alpha_{n} / 2\right\}\right)+\mathbb{P}\left(\left\{\left|f_{n}-g_{n}\right|>\alpha_{n} / 2\right\}\right) \\
& \leq \frac{\beta_{n}}{2}+\frac{1}{\Psi\left(\alpha_{n} / 2 \varepsilon_{n}\right)} \leq \beta_{n} .
\end{aligned}
$$


286 Weak compactness for operators on subspaces of Orlicz spaces

To end the construction, it remains to note that

$$
\begin{aligned}
\left\|f_{n}-\breve{g}_{n}\right\|_{\Psi} & \leq\left\|f_{n}-g_{n}\right\|_{\Psi}+\left\|\breve{g}_{n}-g_{n}\right\|_{\Psi} \leq \varepsilon_{n}+\frac{1}{a}\left\|\breve{g}_{n}-g_{n}\right\|_{\infty} \\
& \leq \frac{1}{2^{n+1}}+\frac{\alpha_{n}}{a} \leq \frac{1}{2^{n}} \leq \frac{1}{2}
\end{aligned}
$$

and so

$$
\left\|\breve{g}_{n}\right\|_{\Psi} \geq\left\|f_{n}\right\|_{\Psi}-\left\|f_{n}-\breve{g}_{n}\right\|_{\Psi} \geq 1-\frac{1}{2}=\frac{1}{2} .
$$

This ends the inductive construction.

Consider now

$$
\breve{g}=\sum_{n=1}^{+\infty}\left|\breve{g}_{n}\right| .
$$

Set $A_{n}=\left\{\left|g_{n}\right|>\alpha_{n}\right\}$ and, for $n \geq 1$

$$
B_{n}=A_{n} \backslash \bigcup_{j>n} A_{j} .
$$

We have $\mathbb{P}\left(\lim \sup A_{n}\right)=0$, because

$$
\sum_{n \geq 1} \mathbb{P}\left(A_{n}\right) \leq \sum_{n \geq 1} \beta_{n} \leq \sum_{n \geq 1} \frac{1}{2^{n}}<+\infty .
$$

Now $\breve{g}$ vanishes out of $\bigcup_{n \geq 1} B_{n} \cup\left(\limsup A_{n}\right)$ and we have

$$
\begin{aligned}
\int_{B_{n}} \Psi\left(\frac{\left|\breve{g}_{n}\right|}{2 M}\right) d \mathbb{P} & \leq \int_{\Omega} \Psi\left(\frac{\left|g_{n}\right|}{2 M}\right) d \mathbb{P} \\
& \leq \int_{\Omega} \Psi\left(\frac{\left|g_{n}-f_{n}\right|}{2 M}+\frac{\left|f_{n}\right|}{2 M}\right) d \mathbb{P} \\
& \leq \frac{1}{2} \int_{\Omega} \Psi\left(\frac{\left|g_{n}-f_{n}\right|}{M}\right) d \mathbb{P}+\frac{1}{2} \int_{\Omega} \Psi\left(\frac{\left|f_{n}\right|}{M}\right) d \mathbb{P} .
\end{aligned}
$$

The first integral is less than $\varepsilon_{n} / M$, because $\Psi(a t) \leq a \Psi(t)$ for $0 \leq a \leq 1$ and $\varepsilon_{n} / M \leq 1$, so that

$$
\int_{\Omega} \Psi\left(\frac{\left|g_{n}-f_{n}\right|}{M}\right) d \mathbb{P} \leq \frac{\varepsilon_{n}}{M} \int_{\Omega} \Psi\left(\frac{\left|g_{n}-f_{n}\right|}{\varepsilon_{n}}\right) d \mathbb{P} \leq \frac{\varepsilon_{n}}{M} \leq \frac{1}{2^{n+1}}
$$

since $\left\|f_{n}-g_{n}\right\|_{\Psi} \leq \varepsilon_{n}$. Since

$$
\int_{\Omega} \Psi\left(\frac{\left|f_{n}\right|}{M}\right) d \mathbb{P} \leq \frac{\beta_{n}}{2} \Psi\left(\alpha_{n} / 2 M\right) \leq \beta_{n} / 2,
$$


we obtain

$$
\int_{B_{n}} \Psi\left(\frac{\left|\breve{g}_{n}\right|}{2 M}\right) d \mathbb{P} \leq \frac{1}{2^{n+2}}+\frac{\beta_{n}}{4} .
$$

Therefore, since $\mathbb{P}\left(B_{n}\right) \leq \mathbb{P}\left(A_{n}\right) \leq \beta_{n}$, we have

$$
\begin{aligned}
\int_{\Omega} \Psi\left(\frac{|\breve{g}|}{4 M}\right) d \mathbb{P} & =\sum_{n=1}^{+\infty} \int_{B_{n}} \Psi\left(\frac{|\breve{g}|}{4 M}\right) d \mathbb{P} \\
& \leq \sum_{n=1}^{+\infty} \int_{B_{n}} \frac{1}{2}\left[\Psi\left(\frac{\left\|g_{1}\right\|_{\infty}+\cdots+\left\|g_{n-1}\right\|_{\infty}}{2 M}\right)+\Psi\left(\frac{\left|\breve{g}_{n}\right|}{2 M}\right)\right] d \mathbb{P}
\end{aligned}
$$

by convexity of $\Psi$ and because $\breve{g}_{j}=0$ on $B_{n}$ for $j>n$

$$
\begin{aligned}
& \leq \frac{1}{2} \sum_{n=1}^{+\infty}\left(M_{n} \beta_{n}+\frac{1}{2^{n+2}}+\frac{\beta_{n}}{4}\right) \\
& \leq \frac{1}{2} \sum_{n=1}^{+\infty}\left(\frac{1}{2^{n+1}}+\frac{1}{2^{n+2}}+\frac{1}{2^{n+2}}\right) \leq 1
\end{aligned}
$$

which proves that $\breve{g} \in L^{\Psi}$, and consequently that the series $\sum_{n \geq 1} \breve{g}_{n}$ is weakly unconditionally Cauchy in $L^{\Psi}$ :

$$
\sup _{n \geq 1} \sup _{\theta_{k}= \pm 1}\left\|\sum_{k=1}^{n} \theta_{k} \breve{g}_{k}\right\|_{\Psi} \leq \sup _{n \geq 1}\left\|\sum_{k=1}^{n}\left|\breve{g}_{k}\right|\right\|_{\Psi} \leq\|\breve{g}\|_{\Psi} \leq 4 M .
$$

Since $\left\|\breve{g}_{n}\right\|_{\psi} \geq 1 / 2,\left(\breve{g}_{n}\right)_{n \geq 1}$ has, by Bessaga-Pełczyński's theorem, a subsequence $\left(\breve{g}_{n_{k}}\right)_{k \geq 1}$ which is equivalent to the canonical basis of $c_{0}$. The corresponding subsequence $\left(f_{n_{k}}\right)_{k \geq 1}$ of $\left(f_{n}\right)_{n \geq 1}$ remains equivalent to the canonical basis of $c_{0}$, since

$$
\sum_{n=1}^{+\infty}\left\|f_{n}-\breve{g}_{n}\right\|_{\Psi} \leq \sum_{n=1}^{+\infty} \varepsilon_{n}+\frac{\alpha_{n}}{a} \leq \sum_{n=1}^{+\infty} \frac{1}{2^{n+1}}+\frac{1}{2^{n+2}}<1
$$

and the assertion follows.

\section{Comments}

Remark 12. Let us note that the assumption $X \subseteq M^{\Psi}$ in Theorem 4 cannot be relaxed in general. In fact, suppose that $X$ is a subspace of $L^{\Psi}$ containing $L^{\infty}$, and let $\xi \in\left(M^{\psi}\right)^{\perp} \subseteq\left(L^{\Psi}\right)^{*}, \xi \neq 0$. Being of rank one, $\xi$ is trivially weakly compact. Suppose that it satisfies (W). Let $f \in X$ with norm 1 , and let $\varepsilon>0$. For $t$ large enough and $f_{t}=f \mathbb{1}_{\{|f| \leq t\}}$, we have $\left\|f-f_{t}\right\|_{2} \leq \varepsilon / C_{\varepsilon}$. Moreover, $f_{t} \in L^{\infty} \subseteq X$ and $\left\|f_{t}\right\|_{\Psi} \leq\|f\|_{\Psi}=1$. Since 
$\xi$ vanishes on $L^{\infty}$ and $f-f_{t} \in X$, we get

$$
|\xi(f)|=\left|\xi\left(f-f_{t}\right)\right| \leq C_{\varepsilon}\left\|f-f_{t}\right\|_{2}+\varepsilon\left\|f-f_{t}\right\|_{\Psi} \leq 3 \varepsilon .
$$

This implies that $\xi(f)=0$. Since this occurs for every $\xi \in\left(M^{\Psi}\right)^{\perp}$, we get that $X \subseteq M^{\Psi}$ (and actually $X=M^{\Psi}$ since $X$ contains $L^{\infty}$ ).

In particular Theorem 4 does not hold for $X=L^{\Psi}$.

Remark 13. However, condition (W) remains true for bi-adjoint operators coming from subspaces of $M^{\Psi}$ : if $T: X \subseteq M^{\Psi} \rightarrow Y$ satisfies the condition (W), then $T^{* *}: X^{* *} \rightarrow Y^{* *}$ also satisfies it. Indeed, for every $\varepsilon>0$, we get an equivalent norm $\||\cdot|\|_{\varepsilon}$ on $X$ by putting

$$
\||f|\|_{\varepsilon}=C_{\varepsilon}\|f\|_{2}+\varepsilon\|f\|_{\Psi} .
$$

Hence if $f \in X^{* *}$, there exists a net $\left(f_{\alpha}\right)_{\alpha}$ of elements in $X$, with $\left\|\left|f_{\alpha}\right|\right\|_{\varepsilon} \leq\||f|\|_{\varepsilon}$ which converges weak-star to $f$. Then $\left(T f_{\alpha}\right)_{\alpha}$ converges weak-star to $T^{* *} f$, and

$$
\begin{aligned}
\left\|T^{* *} f\right\| & \leq \liminf _{\alpha}\left\|T f_{\alpha}\right\| \leq \liminf _{\alpha}\left(C_{\varepsilon}\left\|f_{\alpha}\right\|_{2}+\varepsilon\left\|f_{\alpha}\right\|_{\Psi}\right) \\
& =\liminf _{\alpha}\left\|\left|f_{\alpha}\right|\right\|_{\varepsilon} \leq\|f \mid\|_{\varepsilon}=C_{\varepsilon}\|f\|_{2}+\varepsilon\|f\|_{\Psi} .
\end{aligned}
$$

Hence, from Proposition 10 above, for such a $T, T^{* *}$ is weakly compact if and only if it satisfies (W). We shall use this fact in the forthcoming paper [13].

Remark 14. In Theorem 4, we cannot only assume that $\Psi \notin \Delta_{2}$, instead of $\Psi \in \Delta^{0}$, as the following example shows. It also shows that in Corollary 2, we cannot obtain condition (W) instead of condition (1).

Example 15. Let us define

$$
\psi(t)= \begin{cases}t & \text { for } 0 \leq t<1 \\ (k !)(k+2) t-k !(k+1) ! & \text { for } k ! \leq t \leq(k+1) !, \quad k \geq 1,\end{cases}
$$

$\left(\psi(k !)=(k !)^{2}\right.$ for every integer $k \geq 1$ and $\psi$ is linear between $k !$ and $(k+1) !)$, and $\Psi(x)=\int_{0}^{x} \psi(t) d t$. Since $t^{2} \leq \psi(t)$ for all $t \geq 0$, one has $x^{3} / 3 \leq \Psi(x)$ for all $x \geq 0$. Then

$$
\Psi(2 . n !) \geq \int_{n !}^{2 . n !} \psi(t) d t=n !(n+2) \frac{3}{2}(n !)^{2}-(n !)^{2}(n+1) !=(n !)^{3}\left(\frac{n}{2}+2\right),
$$

whereas

$$
\Psi(n !)=\int_{0}^{n !} \psi(t) d t \leq(n !)^{2} n !=(n !)^{3}
$$


hence

$$
\frac{\Psi(2 . n !)}{\Psi(n !)} \geq \frac{n}{2}+2
$$

and so

$$
\limsup _{x \rightarrow+\infty} \frac{\Psi(2 x)}{\Psi(x)}=+\infty,
$$

which means that $\Psi \notin \Delta_{2}$. On the other hand, for every $\beta>1$

$$
\Psi(n ! / \beta) \geq \frac{1}{3}\left(\frac{n !}{\beta}\right)^{3}=\frac{(n !)^{3}}{3 \beta^{3}},
$$

so

$$
\frac{\Psi(n !)}{\Psi(n ! / \beta)} \leq \frac{(n !)^{3}}{(n !)^{3} / 3 \beta^{3}}=3 \beta^{3}
$$

hence

$$
\liminf _{x \rightarrow+\infty} \frac{\Psi(2 x)}{\Psi(x)} \leq 3 \beta^{3},
$$

and $\Psi \notin \Delta^{0}$ (actually, this will follow too from the fact that Theorem 4 is not valid for this $\Psi)$.

Moreover, the conjugate function of $\Psi$ satisfies the condition $\Delta_{2}$. Indeed, since $\psi$ is convex, one has $\psi(2 u) \geq 2 \psi(u)$ for all $u \geq 0$, and hence:

$$
\Psi(2 x)=\int_{0}^{2 x} \psi(t) d t=2 \int_{0}^{x} \psi(2 u) d u \geq 2 \int_{0}^{x} 2 \psi(u) d u=4 \Psi(x),
$$

and as it was seen in the Introduction that means that $\Psi \in \nabla_{2}$.

Now, we have $x^{3} / 3 \leq \Psi(x)$ for all $x \geq 0$; therefore $\|\cdot\|_{3} \leq 3^{1 / 3}\|\cdot\|_{\Psi}$. In particular, we have an inclusion map $j: M^{\Psi} \hookrightarrow L^{3}$, which is, of course, weakly compact. Nevertheless, assuming that $\mathbb{P}$ is diffuse, condition (W) is not verified by $j$, when $\varepsilon<1$. Indeed, as we have seen before, one has $\Psi(n !) \leq(n !)^{3}$. Hence, if we choose a measurable set $A_{n}$ such that $\mathbb{P}\left(A_{n}\right)=1 / \Psi(n !)$, we have

$$
\left\|\mathbb{I}_{A_{n}}\right\|_{\Psi}=\frac{1}{\Psi^{-1}\left(1 / \mathbb{P}\left(A_{n}\right)\right)}=\frac{1}{n !} ;
$$

whereas

$$
\left\|\mathbb{I}_{A_{n}}\right\|_{3}=\mathbb{P}\left(A_{n}\right)^{1 / 3}=\frac{1}{\Psi(n !)^{1 / 3}} \geq \frac{1}{n !}
$$

and

$$
\left\|\mathbb{I}_{A_{n}}\right\|_{2}=\mathbb{P}\left(A_{n}\right)^{1 / 2} \leq\left[\frac{3}{(n !)^{3}}\right]^{1 / 2}=\frac{\sqrt{3}}{(n !)^{3 / 2}} .
$$


If condition (W) were true, we should have, for every $n \geq 1$

$$
\frac{1}{n !} \leq C_{\varepsilon} \frac{\sqrt{3}}{(n !)^{3 / 2}}+\varepsilon \frac{1}{n !},
$$

that is $\sqrt{n !} \leq \sqrt{3} \frac{C_{\varepsilon}}{1-\varepsilon}$, which is of course impossible for $n$ large enough.

Remark 16. In the case of the whole space $M^{\Psi}$, we can give a direct proof of the necessity in Theorem 4. Indeed, suppose that $T: M^{\Psi} \rightarrow X$ is weakly compact. Then $T^{*}: X^{*} \rightarrow L^{\Phi}=\left(M^{\Psi}\right)^{*}$ is weakly compact, and so the set $K=T^{*}\left(B_{X^{*}}\right)$ is relatively weakly compact.

Since $\Phi$ satisfies the $\Delta^{0}$ condition, it follows from [1] (Corollary 2.9) that $K$ has equi-absolutely continuous norms. Hence, for every $\varepsilon>0$, we can find $\delta_{\varepsilon}>0$ such that:

$$
m(A) \leq \delta_{\varepsilon} \quad \Rightarrow \quad\left\|g \mathbb{I}_{A}\right\|_{\Phi} \leq \varepsilon / 2, \quad g \in T^{*}\left(B_{X^{*}}\right) .
$$

But (the factor 1/2 appears because we use the Luxemburg norm on the dual, and not the Orlicz norm: see [16], Proposition III.3.4)

$$
\begin{aligned}
\sup _{g \in T^{*}\left(B_{X^{*}}\right)}\left\|g \mathbb{I}_{A}\right\|_{\Phi} & \geq \frac{1}{2} \sup _{u \in B_{X^{*}}} \sup _{f \|_{\Psi} \leq 1}\left|<f,\left(T^{*} u\right) \mathbb{I}_{A}>\right| \\
& =\frac{1}{2} \sup _{u \in B_{X^{*}}} \sup _{f \|_{\Psi} \leq 1}\left|\int f\left(T^{*} u\right) \mathbb{I}_{A} d m\right| \\
& =\frac{1}{2} \sup _{u \in B_{X^{*}}} \sup _{f \|_{\Psi} \leq 1}\left|<T\left(f \mathbb{I}_{A}\right), u>\right|=\frac{1}{2} \sup _{\|f\|_{\Psi} \leq 1}\left\|T\left(f \mathbb{I}_{A}\right)\right\| ;
\end{aligned}
$$

so

$$
m(A) \leq \delta_{\varepsilon} \quad \Rightarrow \quad \sup _{\|f\|_{\Psi} \leq 1}\left\|T\left(f \mathbb{1}_{A}\right)\right\| \leq \varepsilon .
$$

Now, we have

$$
m\left(|f| \geq\|f\|_{2} / \delta_{\varepsilon}\right) \leq \frac{\delta_{\varepsilon}}{\|f\|_{2}} \int|f| d m=\frac{\delta_{\varepsilon}}{\|f\|_{2}}\|f\|_{1} \leq \delta_{\varepsilon} ;
$$

hence, with $A=\left\{|f| \geq\|f\|_{2} / \delta_{\varepsilon}\right\}$, we get, for $\|f\|_{\Psi} \leq 1$ :

$$
\|T f\| \leq\left\|T\left(f \mathbb{I}_{A}\right)\right\|+\left\|T\left(f \mathbb{I}_{A^{c}}\right)\right\| \leq \varepsilon+\|T\| \frac{\|f\|_{2}}{\delta_{\varepsilon}}
$$

since $\left|f \mathbb{1}_{A^{c}}\right| \leq\|f\|_{2} / \delta_{\varepsilon}$ implies $\left\|f \mathbb{1}_{A^{c}}\right\|_{\Psi} \leq\left\|f \mathbb{1}_{A^{c}}\right\|_{\infty} \leq\|f\|_{2} / \delta_{\varepsilon}$.

Remark 17. Conversely, E. Lavergne ([10]) recently uses our Theorem 4 to give a proof of the above quoted result of J. Alexopoulos ([1], Corollary 2.9), and uses it to show that, when $\Psi \in \Delta^{0}$, then the reflexive 
subspaces of $L^{\Phi}$ (where $\Phi$ is the conjugate of $\Psi$ ) are closed for the $L^{1}$ norm.

Acknowledgements. This work was made during the stay in Lens as Professeur invité de l'Université d'Artois, in May-June 2005, and a visit in Lens and Lille in March 2006 of the fourth-named author. He would like to thank both Mathematics Departments for their kind hospitality.

\section{References}

[1] J. Alexopoulos, De la Vallée-Poussin's theorem and weakly compact sets in Orlicz spaces, Quaestiones Math., 17 (1994), 231-248.

[2] B. Beauzamy, Propriétés géométriques des espaces d'interpolation, Séminaire Maurey-Schwartz 1974-1975: Espaces $L_{p}$, applications radonifiantes et géométrie des espaces de Banach, Exp. No. XIV, 17 pp., Centre Math., École Polytech., Paris, 1975.

[3] J. Diestel, H. Jarchow and A. Tonge, Absolutely Summing Operators, Cambridge studies in advanced mathematics, 43, Cambridge University Press, 1995.

[4] G. Godefroy and P. Saab, Quelques espaces de Banach ayant les propriétés $(V)$ ou $\left(V^{*}\right)$ de A. Petczyński, C.R. Acad. Sci. Paris, Série A, 303 (1986), 503-506.

[5] G. Godefroy and P. Saab, Weakly unconditionally convergent series in $M$-ideals, Math. Scand., 64 (1989), 307-318.

[6] P. Harmand, D. Werner and W. Werner, $M$-ideals in Banach Spaces and Banach Algebras, Lecture Notes in Math., 1547, Springer, 1993.

[7] H. Jarchow, On Weakly Compact Operators on $\mathbf{C}^{*}$-Algebras, Math. Ann., 273 (1986), 341-343.

[8] H. Jarchow and U. Matter, On weakly compact operators on $\mathcal{C}(K)$ spaces, 80-88, Proceedings of the Missouri Conference held in Columbia, USA, June 24-29 1984, N. Kalton and E. Saab, Eds., Lecture Notes in Math., 1166, 1985.

[9] M. A. Krasnosel'skiı̌ and Ya. B. Rutickiı̌, Convex functions and Orlicz spaces (translation), P. Noordhoff Ltd., Groningen, 1961.

[10] E. Lavergne, Reflexive subspaces of some Orlicz spaces, Colloq. Math., 113 (2008), 333-340.

[11] P. Lefèvre, Some characterizations of weakly compact operators on $H^{\infty}$ and on the disk algebra. Application to composition operators, Journal of Operator Theory, 54 (2005), 229-238. 
292 Weak compactness for operators on subspaces of Orlicz spaces

[12] P. Lefèvre, D. Li, H. Queffélec and L. Rodríguez-Piazza, Some translation-invariant Banach function spaces which contain $c_{0}$, Studia Math., 163 (2004), 137-155.

[13] P. Lefèvre, D. Li, H. Queffélec and L. Rodríguez-Piazza, Composition operators on Hardy-Orlicz spaces, Memoirs Amer. Math. Soc., to appear.

[14] C. P. Niculescu, Absolute continuity and weak compactness, Bull. Amer. Math. Soc., 81 (1975), 1064-1066.

[15] C. P. Niculescu, Absolute continuity in Banach space theory, Rev. Roum. Math. Pures Appl., 24 (1979), 413-422.

[16] M. M. Rao and Z. D. Ren, Theory of Orlicz Spaces, Pure and Applied Mathematics 146, Marcel Dekker, Inc., 1991.

[17] D. Werner, New classes of Banach spaces which are $M$-ideals in their biduals, Math. Proc. Cambridge Philos. Soc., 111 (1992), 337-354.

P. Lefèvre and D. Li

Université d'Artois

Laboratoire de Mathématiques de Lens EA 2462

Fédération CNRS Nord-Pas-de-Calais FR 2956

Faculté des Sciences Jean Perrin, Rue Jean Souvraz, S.P.18

62307 LENS Cedex

FRANCE

(E-mail : pascal.lefevre@euler.univ-artois.fr)

(E-mail : daniel.li@euler.univ-artois.fr)

H. Queffélec

Université des Sciences et Technologies de Lille

Laboratoire Paul Painlevé U.M.R. CNRS 8524

U.F.R. de Mathématiques,

59655 VILLENEUVE D’ASCQ Cedex

FRANCE

(E-mail : queff@math.univ-lille1.fr)

Luis Rodríguez-Piazza

Universidad de Sevilla

Facultad de Matemáticas, Dpto de Análisis Matemático

Apartado de Correos 1160

41080 SEVILLA

SPAIN

(E-mail : piazza@us.es)

(Received : September 2007) 


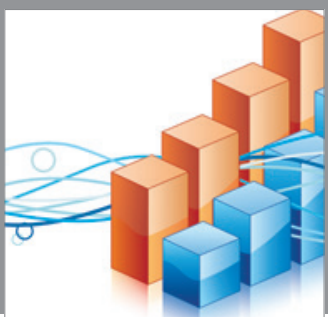

Advances in

Operations Research

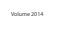

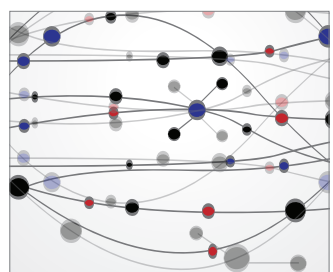

\section{The Scientific} World Journal
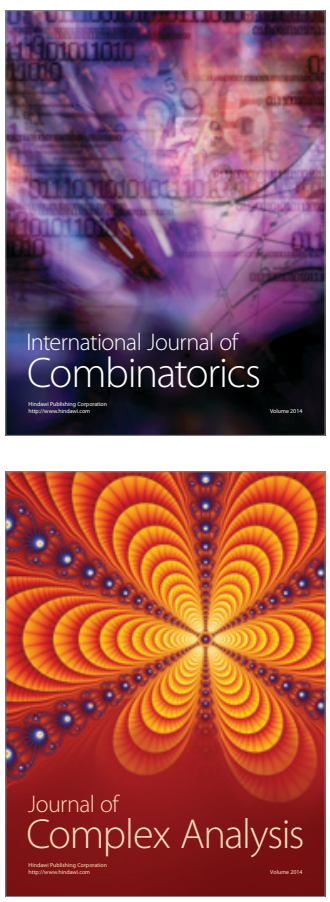

International Journal of

Mathematics and

Mathematical

Sciences
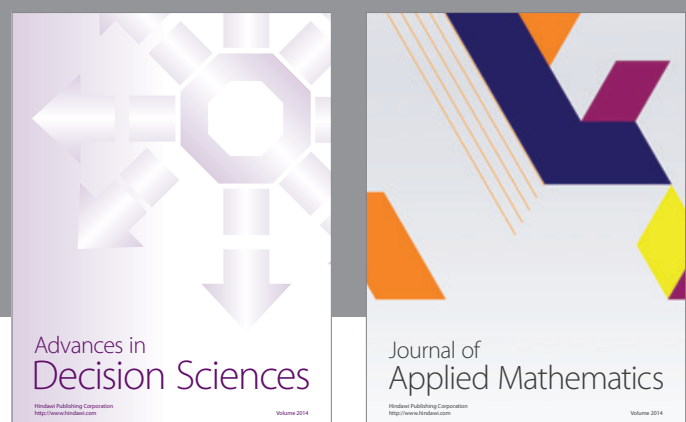

Journal of

Applied Mathematics
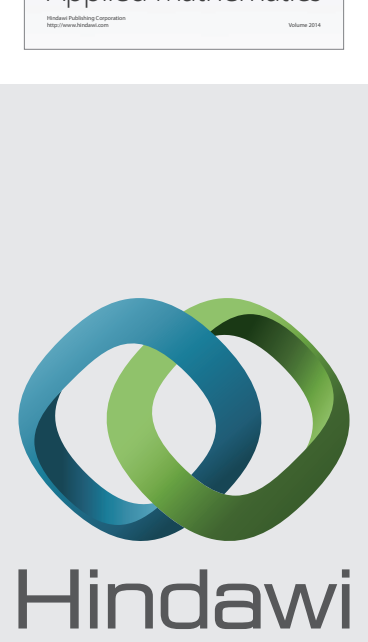

Submit your manuscripts at http://www.hindawi.com
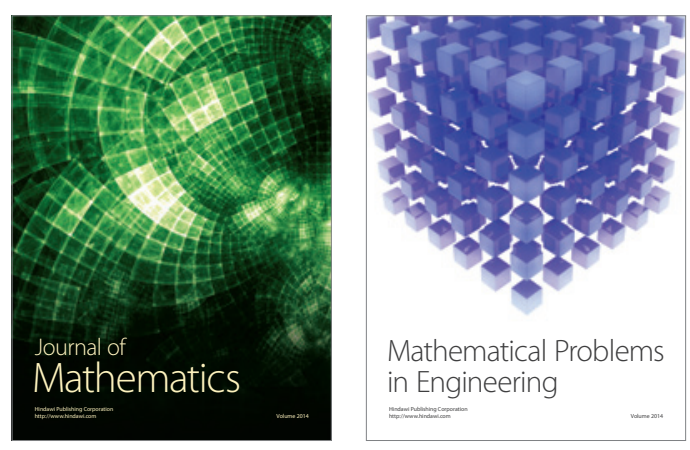

Mathematical Problems in Engineering
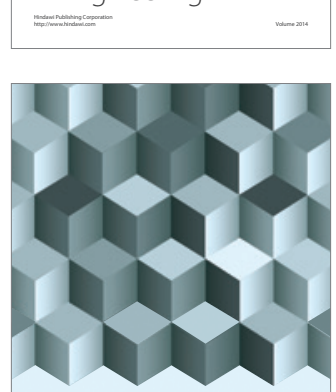

Journal of

Function Spaces
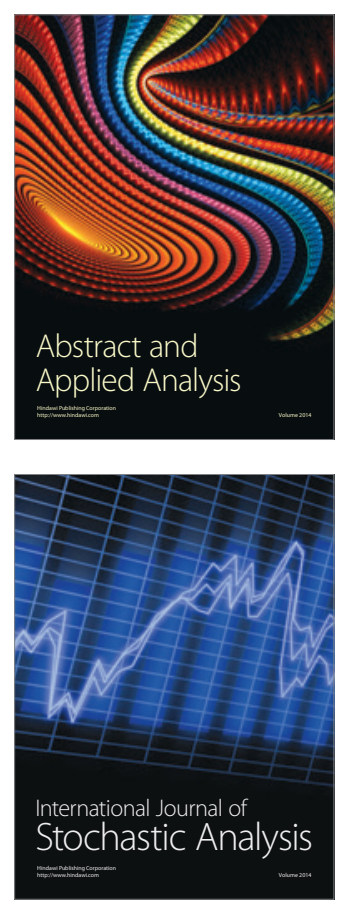

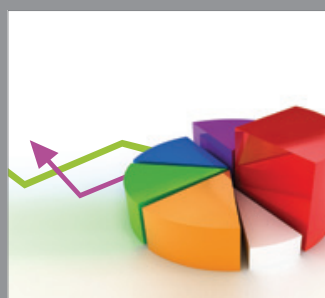

ournal of

Probability and Statistics

Promensencen
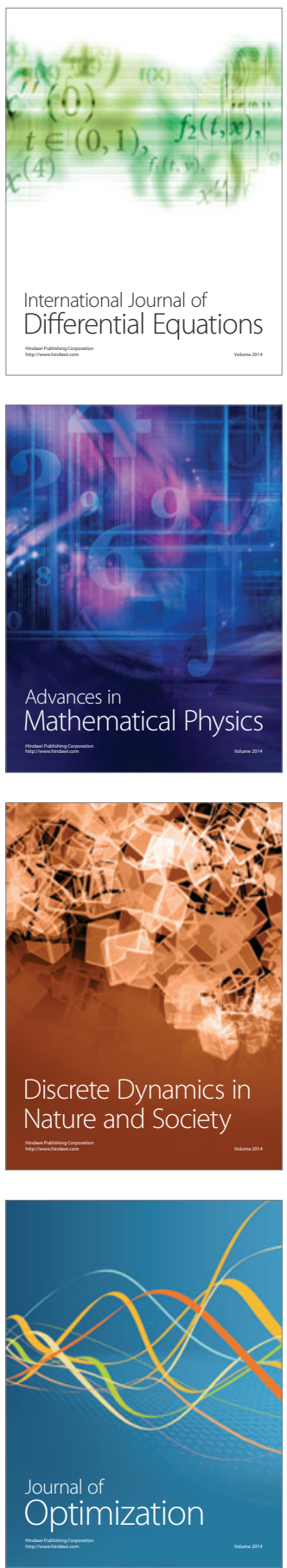